\title{
Process rather than pattern: finding pine needles in the coevolutionary haystack David R Nash
}

Address: Centre for Social Evolution, Department of Biology, University of Copenhagen, Universitetsparken 15, DK-2100 Copenhagen, Denmark. E-mail: DRNash@bio.ku.dk

Published: 28 May 2008

Journal of Biology 2008, 7:14 (doi:10.1 186/jbiol75)

The electronic version of this article is the complete one and can be found online at http://jbiol.com/content/7/5/14

(c) 2008 BioMed Central Ltd

\begin{abstract}
The geographic mosaic theory is fast becoming a unifying framework for coevolutionary studies. A recent experimental study of interactions between pines and mycorrhizal fungi in $B M C$ Biology is the first to rigorously test geographical selection mosaics, one of the cornerstones of the theory.
\end{abstract}

Coevolution is a powerful concept in biology. It explains why cheetahs run fast, and why gazelles run fast too [1]. It explains why the flowers of some orchids have extraordinarily long spurs to store their nectar, and why the moths that pollinate them have extraordinarily long tongues to drink it [2]. It explains why we don't all succumb to diseases, and why diseases still exist [3]. Most evolutionary change may well be coevolutionary change [4].

How coevolution actually works is far from clear, however, if one looks into it in any depth. How is the genetic variation that is the raw material of coevolution, or any other sort of evolution for that matter, maintained when faster or longer is always better? How can genetically homogeneous populations attacked by pathogens survive long enough to mount a coevolutionary response? The answers may lie in the fact that the world is not made up of single populations of organisms freely exchanging genes. Instead, populations are not the same everywhere, and interactions between organisms are not the same everywhere. As is clear from our own species' experience with its coevolving pathogens [5], things vary geographically.

It was thinking about such questions over the past two decades that led John Thompson to propose his geographic mosaic theory of coevolution [6,7]. Although this theory has been widely discussed and has become a unifying framework for many coevolutionary studies, it is still often misunderstood [8]. That there are differences between how organisms interact at different spots on Earth is, in itself, a fairly trivial observation, but Thompson's big idea is that without those differences, there would be no coevolution. The geographic mosaic drives coevolution, rather than being merely a consequence of the fragmentation of interacting populations. Pattern and process are quite distinct in the theory, but they are often confused in practice. It is significant that Thompson's book The Coevolutionary Process [6], which first brought his theory to most of the scientific community, emphasizes process rather than pattern. The processes underlying the geographic mosaic theory of coevolution are difficult to test experimentally, but a new study in BMC Biology [9] of pines and their mycorrhizal fungi, provides the first experimental support for one of the key processes.

\section{Processes}

The geographic mosaic theory of coevolution puts forward three distinct processes that are conjectured to be the basis of coevolutionary change (Figure 1): coevolutionary hot and cold spots, selection mosaics and trait-mixing. 


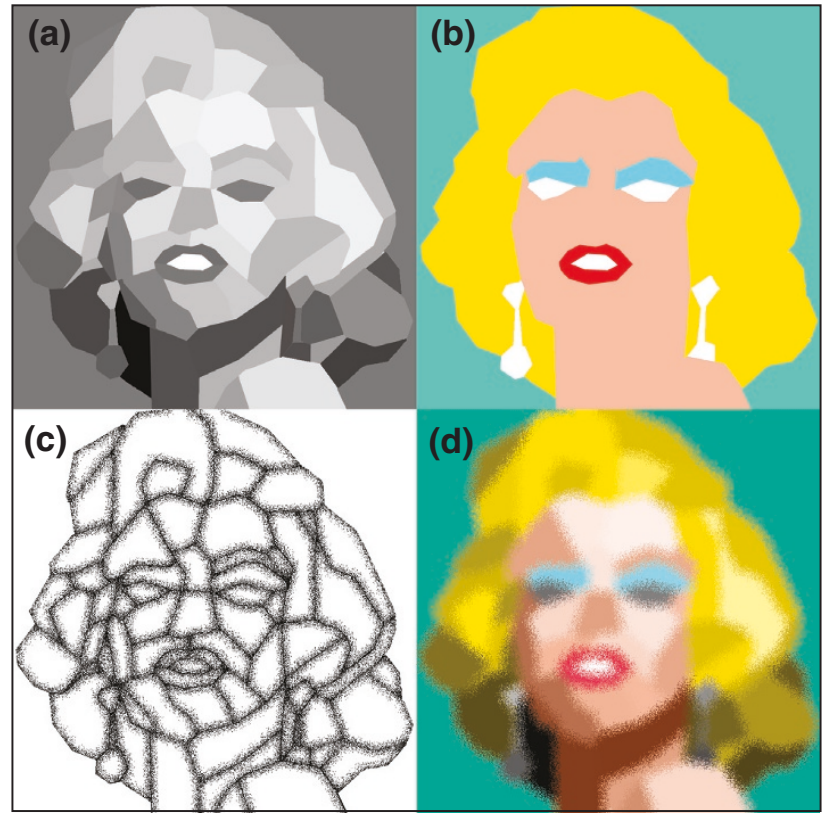

Figure I

The three components of the geographic mosaic of coevolution. (a) Populations of interacting species are distributed in a spatial mosaic, with the strength of coevolutionary selection exerted by each partner on the other varying between populations. In cold spots (here represented by light-colored tiles), the traits of each species evolve independently, whereas in hot spots (dark tiles) coevolutionary selection is intense. (b) As well as varying in strength, the direction of selection varies spatially (there is a selection mosaic; represented here as different colored tiles), depending on the interactions between the genotypes of both interacting species and the local environment. (c) There is some mixing of genes due to the dispersal of individuals between populations (represented as the individual dots making up the shaded areas). The level of mixing must be sufficient to allow the occasional introduction of new genotypes into populations, but low enough that adaptations are not swamped by gene flow from populations experiencing different selection pressures or strengths. (d) The combination of all three elements leads to a system in which coevolution is a continuous dynamic process that, at the same time, retains ample genetic variation to allow long-term coevolution.

The strength of coevolution varies between populations of interacting organisms. In some areas, termed hot spots, coevolutionary selection is intense, whereas in cold spots, the interacting species evolve independently of each other [10]. This can be for the simple reason that one of the interacting partners is absent in a cold spot, as is often the case with parasitic interactions in which not every host population is parasitized $[11,12]$. Cold spots can also exist for other reasons, for example because alternative hosts are present that are preferred by a parasite [13]. There is a continuum between cold and hot spots, with the strength of coevolutionary selection increasing as spots become progressively 'hotter'.
Selection mosaics are also important for the theory, but these have often been misunderstood. It is not enough that the strength of coevolutionary selection varies between populations, it is also necessary that the direction of that selection varies, so that the outcomes of coevolution are different in different populations, depending on their environment. In other words, the costs and benefits to both partners of any particular adaptation are dependent not only on the adaptations of their partner, but also on the environment in which the interaction occurs. This is perhaps most easily seen in what have been termed 'conditional mutualisms' [14], in which interactions can be mutualistic, commensal or parasitic depending on the ecological conditions in which the partners interact [15-17]. The variation does not, however, need to be so great as to lead to shifts between parasitism and mutualism, but outcomes are dependent on the interactions of the adaptations of both partners with the environment that they find themselves in. Hence, the selection mosaic is a result of gene $x$ gene $x$ environment $(G \times G \times E)$ interactions $[8,18]$. The different outcomes in different environments can be due either to abiotic factors [19] or to biotic factors, such as the presence and density of a predator or competitor $[13,20,21]$.

Finally, in order for the coevolutionary process to work, there must be a mechanism that allows traits that have evolved in one population to be transferred to and mixed with traits that have evolved in other areas. In other words, there must be gene flow between populations to enable genes that are favorable to track the conditions in which they are favorable, and to allow the maintenance of genetic variation that would otherwise disappear [11,22]. Gene flow must also be at the right level; too much mixing, and there will never be a response to selection because the best adapted genes are always swamped by the inflow of nonadaptive genes; too little mixing, however, will allow specific alleles to go to fixation so that, barring novel mutations, the coevolutionary process will grind to a halt $[13,23,24]$.

\section{Patterns}

As well as the three processes involved in coevolutionary mosaics, there are several patterns that are expected to result from the process. For example, it is expected that there will be spatial variation in the traits that are involved with interspecific interactions [25]; that in some areas, traits will be mismatched (local maladaptation) [26]; and finally that there will be few species-level traits that have become fixed as a result of coevolution [27]. These patterns are often used as evidence for the presence of a geographic mosaic of coevolution, but they can also result from other, non-coevolutionary processes. In a key paper last year, Richard Gomul- 


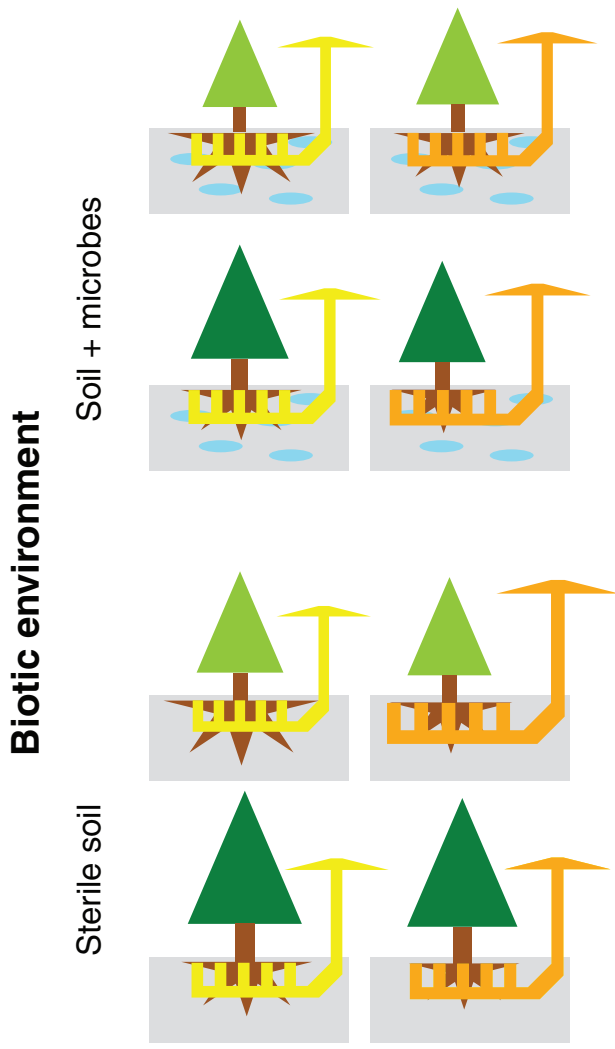

Lab soil
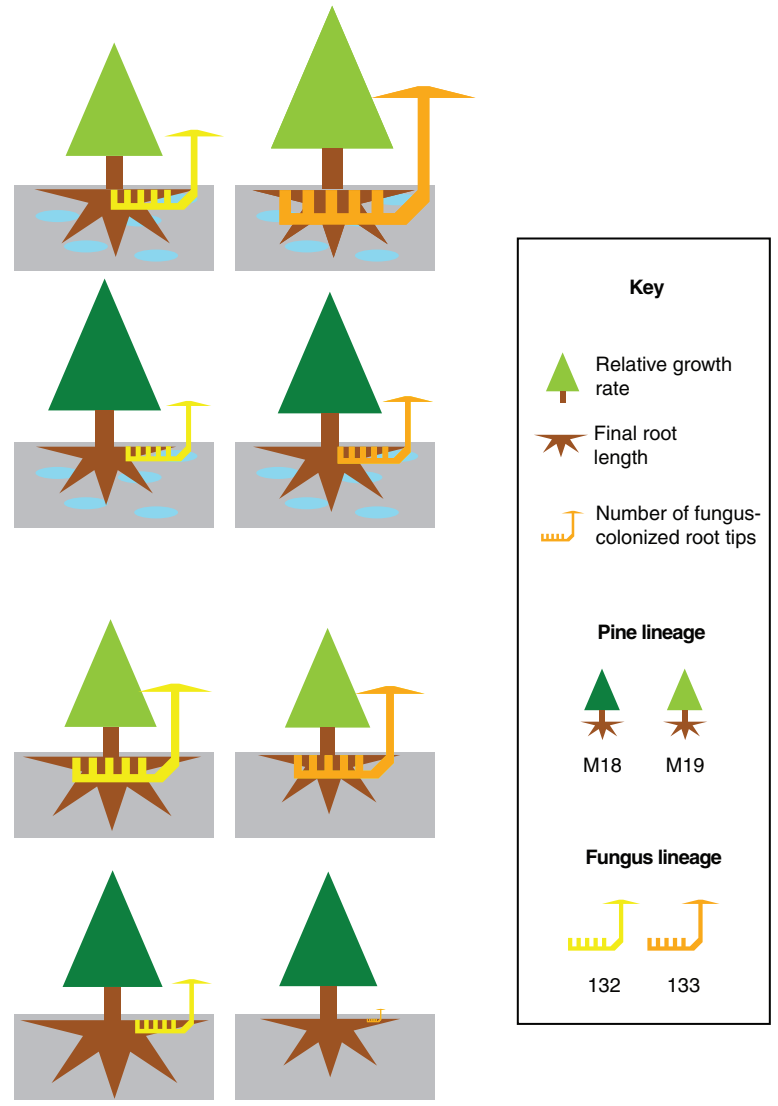

Field soil

Abiotic environment

Figure 2

Summary of the findings of Piculell et al. [9], showing the measured fitness components of two maternal half-sib families of bishop pine plants (MI8 and MI9, measured as relative growth rate and root length) and two full-sib families of its mycorrhizal fungus (I32 and I33, measured as the number of roots of the host that are inoculated) under four different environments. The height of each symbol is proportional to the measured performance value. The performance of both partners in the interaction varies depending on both the lineage of partner they are interacting with and the environment. This is most clearly seen for fungal performance in field soil, where the number of host roots inoculated varies by an order of magnitude.

kiewicz and co-workers [8] set out a rather daunting manifesto for how the geographic mosaic theory of coevolution should be tested and, specifically, how the presence of geographic mosaics of coevolution can be demonstrated. So far there are no studies that have fulfilled all the requirements that have been set forth for testing the theory.

\section{Testing the processes of the coevolutionary mosaic}

There have been several studies inferring hot and cold spots of coevolutionary selection $[11,13,28,29]$, and others characterizing gene flow between populations involved in interspecific interactions $[13,30,31]$, but most studies have been observational rather than experimental, so that process and pattern cannot be disentangled. The area of the theory to which this limitation applies most is the demonstration of selection mosaics, and as a result these have received little rigorous attention. Resolving this deficiency has been the focus of the paper in BMC Biology [9], which is the first to examine explicitly the $G \times G \times E$ interactions required for a selection mosaic to generate coevolutionary change.

The study system chosen was the interaction between bishop pine (Pinus muricata) and the ectomycorrhizal fungus Rhizopogon occidentalis. Interactions between plants and mycorrhizae are strong candidates for model systems to test the 
geographic mosaic theory of coevolution, because there are several clear cases of conditional mutualism in which not only the magnitude but also the nature of interaction (mutualistic or antagonistic) varies between different ecological situations [32-34].

In a simple but elegant factorial experimental design, Piculell et al. [9] tested the interaction between two different lineages of pine and two lineages of fungus in four environments, representing a factorial combination of two different abiotic environments (two different sterile soil types) and two different biotic environments (the presence or absence of potentially competing soil microorganisms). Measuring various fitness components of the pines and fungi showed that there were variable outcomes for the same combinations of pine and fungus lineages under different conditions (Figure 2) and that for one of the pine families, this could indeed result in a mutualistic or parasitic interaction depending on the environment [9].

So, why have such studies not been carried out before? One simple answer is that the need for such studies has only become apparent recently. Another problem is the scale of experimental manipulation required for such studies. Piculell and co-workers [9] needed to successfully raise 128 combinations of pine and fungus, and this was still not quite sufficient to detect any statistically significant $G \times G \times$ E effects (although the $G \times G \times$ biotic environment effects on relative growth rate and shoot:root ratio were close; $P=$ 0.066 and $P=0.059$ respectively; see Additional file 2 in [9]). In other systems, in which changes in interaction strength and direction are likely to be more subtle, the experimental replication required for tests powerful enough to demonstrate selection mosaics is intimidating.

So, although theoretical studies of the geographic mosaic theory of coevolution are multiplying, it is almost inevitable that empirical studies are lagging behind and tend to be concerned with confirming the predicted patterns rather than experimentally testing the process. Translating the outcomes of experimental studies such as that of Piculell et al. [9] into real-world coevolutionary mosaics at the appropriate geographic scale remains a distant goal. In the meantime, large-scale studies of geographical patterns are still crucial for solidifying the foundations of the theory, and for parameterizing the next generation of theoretical models.

\section{Acknowledgements}

I thank Koos Boomsma for valuable discussion and comments. Funding for the Centre for Social Evolution is provided by the Danish National Research Foundation.

\section{References}

I. Dawkins R: River Out of Eden: A Darwinian View of Life. New York: Basic Books; 1995.

2. Darwin C: The Various Contrivances by which Orchids are Fertilised by Insects (2nd, revised edition). London: John Murray; 1877.

3. Haldane JBS: Disease and evolution. La Ricerca Scientifica 1949, 19: 3-10.

4. Van Valen L: How pervasive is coevolution? In Coevolution. Edited by Nitecki MH. Chicago: University of Chicago Press; 1983: I-19.

5. Diamond JM: Guns, Germs and Steel: The Fates of Human Societies. London: Jonathan Cape; 1997.

6. Thompson JN: The Coevolutionary Process. Chicago: University of Chicago Press; 1994.

7. Thompson JN: The Geographic Mosaic of Coevolution. Chicago: University of Chicago Press; 2005.

8. Gomulkiewicz R, Drown DM, Dybdahl MF, Godsoe W, Nuismer SL, Pepin KM, Ridenhour BJ, Smith Cl, Yoder JB: Dos and don'ts of testing the geographic mosaic theory of coevolution. Heredity 2007, 98:249-258.

9. Piculell B, Hoeksema JD, Thompson JN: Interactions of biotic and abiotic environmental factors on an ectomycorrhizal symbiosis, and the potential for selection mosaics. BMC Biol 2008, 6:23.

10. Gomulkiewicz R, Thompson JN, Holt RD, Nuismer SL, Hochberg ME: Hot spots, cold spots, and the geographic mosaic theory of coevolution. Am Nat 2000, 156:156-174.

II. Brockhurst MA, Buckling A, Poullain V, Hochberg ME: The impact of migration from parasite-free patches on antagonistic host-parasite coevolution. Evolution 2007, 61:1238-1243.

12. Nuismer SL, Thompson JN, Gomulkiewicz R: Coevolution between hosts and parasites with partially overlapping geographic ranges. J Evol Biol 2003, 16: I337-1345.

13. Nash DR, Als TD, Maile R, Jones GR, Boomsma J]: A mosaic of chemical coevolution in a large blue butterfly. Science 2008, 319:88-90.

14. Cushman JH, Whitham TG: Conditional mutualism in a membracid-ant association: Temporal, age-specific, and density-dependent effects. Ecology 1989, 70:1040-1047.

15. Offenberg J: Balancing between mutualism and exploitation: the symbiotic interaction between Lasius ants and aphids. Behav Ecol Sociobiol 200I, 49:304-3 I0.

16. van Ommeren RJ, Whitham TG: Changes in interactions between juniper and mistletoe mediated by shared avian frugivores: parasitism to potential mutualism. Oecologia 2002, |30:28|-288.

17. Styrsky JD, Eubanks MD: Ecological consequences of interactions between ants and honeydew-producing insects. Proc Biol Sci 2007, 274: $15 I-164$.

18. Wade MJ: The co-evolutionary genetics of ecological communities. Nat Rev Genet 2007, 8:185-195.

19. Kersch MF, Fonseca CR: Abiotic factors and the conditional outcome of an ant-plant mutualism. Ecology 2005, 86:2 I I7-2I 26.

20. Gaume L, McKey D, Terrin S: Ant-plant-homopteran mutualism: how the third partner affects the interaction between a plant-specialist ant and its myrmecophyte host. Proc Biol Sci 1998, 265:569575.

21. Benkman CW, Holimon WC, Smith JW: The influence of a competitor on the geographic mosaic of coevolution between crossbills and lodgepole pine. Evolution 200I, 55:282-294.

22. Nuismer SL, Thompson JN, Gomulkiewicz R: Gene flow and geographically structured coevolution. Proc Biol Sci 1999, 266:605609.

23. Anderson B, Olivieri I, Lourmas M, Stewart BA: Comparative population genetic structures and local adaptation of two mutualists. Evolution 2004, 58: 1730-1747.

24. Dupas S, Carton Y, Poirie M: Genetic dimension of the coevolution of virulence-resistance in Drosophila - parasitoid wasp relationships. Heredity 2003, 90:84-89.

25. Alcantara JM, Rey PJ, Manzaneda AJ, Boulay R, Ramirez JM, Fedriani JM: Geographic variation in the adaptive landscape for seed size at dispersal in the myrmecochorous Helleborus foetidus. Evol Ecol 2007, 2I:4 I I-430.

26. Thompson JN, Nuismer SL, Gomulkiewicz R: Coevolution and maladaptation. Integr Comp Biol 2002, 42:38I-387. 
27. Thompson JN: Specific hypotheses on the geographic mosaic of coevolution. Am Nat 1999, I53:SI-SI4.

28. Benkman CW: The selection mosaic and diversifying coevolution between crossbills and lodgepole pine. Am Nat 1999, I53:S75-S9I.

29. Brodie ED, Ridenhour BJ: The evolutionary response of predators to dangerous prey: hotspots and coldspots in the geographic mosaic of coevolution between garter snakes and newts. Evolution 2002, 56:2067-2082.

30. Brandt M, Fischer-Blass B, Heinze J, Foitzik S: Population structure and the co-evolution between social parasites and their hosts. Mol Ecol 2007, 16:2063-2078.

31. Martin-Galvez D, Soler J], Martinez JG, Krupa AP, Soler M, Burke $\mathrm{T}$ : Cuckoo parasitism and productivity in different magpie subpopulations predict frequencies of the 457bp allele: a mosaic of coevolution at a small geographic scale. Evolution 2007, 61:2340-2348.

32. Egger KN, Hibbett DS: The evolutionary implications of exploitation in mycorrhizas. Can J Bot 2004, 82: I I 10-I I II.

33. Hoeksema JD, Thompson JN: Geographic structure in a widespread plant-mycorrhizal interaction: pines and false truffles. J Evol Biol 2007, 20: I | 48- I |63.

34. Kiers ET, Lovelock CE, Krueger EL, Herre EA: Differential effects of tropical arbuscular mycorrhizal fungal inocula on root colonization and tree seedling growth: implications for tropical forest diversity. Ecol Lett 2000, 3:106-II3. 